\title{
Las mujeres son cuerpo: reflexiones feministas
}

\author{
Luisa POSADA KUBISSA \\ Universidad Complutense de Madrid \\ lposada@filos.ucm.es
}

Recibido: Mayo 2015

Aceptado: Junio 2015

\section{RESUMEN}

Este artículo se asoma a la concepción del cuerpo y, en particular, de cómo ha sido asociado a lo femenino. A partir de una revisión de las tesis sobre el cuerpo como construcción, en el sentido de Foucault, Bourdieu o Butler, se remite al trabajo actual de la antropóloga española Mari Luz Esteban. Se propone, desde ahí, reivindicar el cuerpo femenino, no como esencia ni como puro constructo, sino como práctica que permite pensar desde un lugar fuera de los lazos mercantilistas y de dominación.

Palabras clave: Cuerpo, constructivismo, sexualización, itinerarios corporales

\section{Women are body: feminist reflections}

\begin{abstract}
This paper deals with the conception of the body paying particular attention to how it has been associated with the feminine. Starting from a revision of theories on the body as a social construction, as argued by Foucault, Bourdieu or Butler, the text highlights the current work of Spanish anthropologist Mari Luz Esteban. Having her work as a starting point, our proposal is to vindicate the female body, neither as essence nor as pure construction, but as a practice that allows us to think of it excluded from "commercial circulation" or domination ties.
\end{abstract}

Key words: Body, constructivism, sexualization, corporal itineraries

\section{EL “CUERPO” DEL CUERPO}

“Las mujeres son cuerpos, y lo son más que el hombre, incitado al éxito social, a la sublimación” (Cixous, 1995: 58).

Como ya lo analizara Simone de Beauvoir en El segundo sexo, la tradición de pensamiento ha vinculado a la mujer a la inmanencia, a la naturaleza, a la reproducción, a la carencia de transcendencia; es decir, al cuerpo (Beauvoir, 1995: 58).Y el cuerpo, como lugar de la identidad femenina, ha venido a ser revalorizado por el pensamiento de la diferencia sexual a finales del siglo XX y, en particular, por Luce Irigaray. 
Resumiendo mucho las propuestas de esta pensadora francesa, diremos que parte del significado de la diferencia en la filosofía postestructuralista del filósofo nietzscheano Jean Deleuze, así como de otros filósofos franceses y postmodernos, como es Jacques Derrida. En estos pensadores, la acepción de lo diferente, de lo que no ha sido asimilado a lo que llaman el discurso logocéntrico- que es el discurso de la razón occidental dominante-, viene a resignificarse, no como "lo inferior", sino como "lo-otro", como "lo no-idéntico", y desde ahí, viene a ser revalorizado. Irigaray parte de que la mujer, en la tradición cultural, ha constituido precisamente ese "lo -otro", esa "diferencia" irreductible a un discurso dominante, "logo-falo-céntrico", que la ha excluido. Y, por esa exclusión, la mujer y su cuerpo, como "diferencia”, representan lo que la propia Irigaray enuncia como "el fleco ciego del logocentrismo".

Una lectura desde el feminismo marxista, como la que realiza Silvia Federici en Calibán y la bruja, interpreta, por otra parte, la expropiación del cuerpo femenino como fenómeno paralelo a la expropiación de las tierras comunales en la transición al capitalismo y sus formas violentas de instauración, en lo que Marx denominó la acumulación originaria (Federici, 2010). Dice Federici:

“¿De qué manera se relacionan la expropiación y la pauperización con el permanente ataque contra las mujeres? ¿Qué podemos aprender acerca del despliegue capitalista, pasado y presente, cuando es examinado desde una perspectiva feminista? Con estas preguntas en mente he vuelto a analizar la «transición» del feudalismo al capitalismo desde el punto de vista de las mujeres, el cuerpo y la acumulación primitiva” (Federici, 2010: 22)

Desde esta lectura podríamos concluir que hay que recuperar el cuerpo, el lugar de la naturaleza, los vínculos comunales e identitarios que están inscritos como significado en la corporalidad de las mujeres. Y si extraemos esta consecuencia no andaríamos muy lejos del discurso de la diferencia sexual, en particular del que hace de la morfología corporal el lugar de la diferencia femenina por excelencia, como es el caso de la propia Irigaray. Y tampoco estaríamos alejadas de esa eticidad hegeliana que asigna a las mujeres el despliegue de una ética movida por lazos privados y familiares y nunca por los más elevados designios de la moralidad.

Pero si lo que se propone es revalorizar el cuerpo femenino, habrá que preguntarse qué se está revalorizando. Porque cabe preguntarse qué es eso del "cuerpo" más allá de cómo ha sido pensado o, por mejor decirlo, de cómo ha sido impuesto:

"En este sentido, lo que constituye el carácter fijo del cuerpo, sus contornos, sus movimientos, será plenamente material, pero la materialidad deberá reconcebirse como el efecto del poder, como el efecto más productivo del poder. Y no habrá modo de interpretar el "género" como una construcción cultural que se impone sobre la superficie de la materia, entendida o bien como "el cuerpo" o bien como su sexo dado. Antes bien, una vez que se entiende el "sexo" mismo en su normatividad, 
la materialidad del cuerpo ya no puede concebirse independientemente de la materialidad de esa norma reguladora” (Butler, 2003: 18-19).

Así entendido, las preguntas son inmediatas: ¿de qué hablamos al hablar del cuerpo?, ¿a qué tipo de realidad prediscursiva hacemos referencia?, ¿cómo va a haber habido cuerpo antes del "cuerpo" constituido a partir de y por las normas biológicas, políticas, culturales?

La modernidad cartesiana despreció el cuerpo, dando prioridad al alma como fuente realmente activa e informada por la divinidad. Como lo lee Federici

“(...) esta batalla contra el cuerpo, caracterizó la época temprana del desarrollo capitalista y que ha continuado, de distintas maneras, hasta nuestros días. De ahí la mecanización del cuerpo, que fue el proyecto de la nueva Filosofía Natural y el punto focal de los primeros experimentos en la organización del Estado” (Federici, 2010: 216).

Pero este desprestigio del cuerpo ya tenía antecedentes lejanos: baste recordar que en el Fedón Platón habla del cuerpo como cárcel del alma. Y tampoco el pensamiento de la Edad Media fue muy afecto a ensalzar la dimensión corporal del ser humano1.

La asociación de la mujer con la corporalidad, su conceptualización como esencialmente cuerpo, permitió concebirla siempre, con diferentes expresiones históricas, como ese lado oscuro e irracional de lo humano, al que la razón debía controlar. Simone de Beauvoir vio claramente que en esta nada inocente dualidad radicaba, en gran medida, la situación inferiorizada de las mujeres. Así lo expresa en El segundo sexo, cuando, al hablar de Los hechos y los mitos, escribe: “¿La mujer? Es muy sencillo, dicen los aficionados a las fórmulas simplistas: es una matriz, un ovario; es una hembra, y basta esa palabra para definirla. En boca del hombre el epíteto "hembra” suena como un insulto" (Beauvoir, 1995: 29).

Resignificar ese "insulto" y hacer valer la corporalidad femenina en positivo ha sido una de las ideas-fuerza del pensamiento de la diferencia sexual. Especialmente del pensamiento fundacional de la diferencia y, en particular, del caso francés. Así, Hélene Cixous apuesta por una escritura que hable con la voz corporalizada, que hable con el cuerpo, y que hable, por tanto, con la diferencia femenina: el cuerpo femenino es concebido ahora como lo que siempre se ha confinado al silencio y, precisamente por ello, este cuerpo ha devenido en lenguaje, en texto. Se trata de un cuerpo desmembrado, como desmembrado está el texto a partir de la postmoderna asunción de la diferencia: de nuevo la revalorización de la mujer como cuerpo, orientada esta vez

${ }^{1}$ Para un tratamiento exhaustivo del tema del cuerpo y su conceptualización en diferentes periodos históricos cabe remitirse a los tres volúmenes de Nadaff, Ramona; Tazi, Nadia; y Feher, Michel (coords.) (1992), Fragmentos para una historia del cuerpo humano, Taurus Ediciones. 
por la escritura que le sirve de salida del discurso de la razón masculina dominante, del discurso falogocéntrico (Cixous, 1995). Para el pensamiento feminista, desde esta posición, el cuerpo es importante, porque es el punto de intersección entre lo físico, lo simbólico y lo material (Reverter, 2001).

Este punto de intersección que es el cuerpo por supuesto lo es en tanto que producto de relaciones y discursos de poder:

"El cuerpo humano entra en un mecanismo de poder que lo explora, lo desarticula y lo recompone. Una 'anatomía política', que es igualmente una 'mecánica del poder' está naciendo; define cómo se puede hacer presa en el cuerpo de los demás, no simplemente para que ellos hagan lo que se desea, sino para que operen como quiere, con las técnicas, según la rapidez y la eficacia que se determina. La disciplina fabrica así cuerpos sometidos y ejercitados, cuerpos 'dóciles' “ (Foucault, 1986: 141).

Pero, la percepción del cuerpo varía en razón de la posición social de dominado o dominante desde la que se aprehende, sobre todo si tenemos en cuenta que

"La imagen social de su cuerpo, con la que cada agente tiene que contar, sin duda desde muy temprano, se obtiene por tanto mediante la aplicación de una taxonomía social, cuyo principio coincide con el de los cuerpos a los que se aplica. Así pues, la mirada no es un mero poder universal y abstracto de objetivación, como pretende Sartre; es un poder simbólico cuya eficacia depende de la posición relativa del que percibe y del que es percibido o del grado en que los esquemas de percepción y de apreciación practicados son conocidos y reconocidos por aquel al que se aplican" (Bourdieu, 2000: 85).

Los conceptos de campo y habitus de Bourdieu permiten atraer estos dos modos de existencia de lo social: el campo como lo social hecho cosa (lo objetivo) y el habitus como lo social inscripto en el cuerpo (lo subjetivo). Las prácticas sociales que ejecutan los agentes se revelan a partir de la relación dialéctica que existe entre ambos (Barrera Sánchez, 2011: 127).

Para examinar la dinámica social es necesario advertir el campo como el lugar en el cual se juegan las posiciones relativas que ocupan los distintos grupos o clases y las relaciones que entre los mismos se establecen y, al mismo tiempo, comprender las formas de conformación de la subjetividad, es decir, la constitución del habitus. Una primera aproximación a este concepto nos permite definirlo como un conjunto de disposiciones duraderas que determinan nuestra forma de actuar, sentir o pensar.

El sujeto vive así su cuerpo, socialmente destinado, como proyecto propio y no como resultado de la lógica social. En sus conclusiones al análisis del dispositivo de sexualidad, Foucault afirma que "hay que decir que existe una sexualidad burguesa, que existen sexualidades de clase”; y abunda en cómo "en el curso del siglo XIX hubo, 
pues, una generalización del dispositivo de sexualidad a partir de un foco hegemónico" (Foucault, 1978: 136). Hablará poco después del cuerpo de las mujeres, al referirse a que
“(...) en el proceso de histerización de la mujer, el "sexo" fue definido de tres maneras: como lo que es común al hombre y la mujer; o como lo que pertenece por excelencia al hombre y falta por lo tanto a la mujer; pero también como lo que constituye por sí solo el cuerpo de la mujer, orientándolo por entero a las funciones de reproducción y perturbándolo sin cesar en virtud de esas mismas funciones" (Foucault, 1978: 162).

Así, el cuerpo femenino va indisociablemente unido, al menos en una de sus definiciones, a la histerización. Y la histerización representa precisamente lo irracional, el pulso ciego, lo que no puede adecuarse a razón, porque es "relativo al útero",2. De modo que las "funciones de la reproducción" orientan y perturban a la vez el cuerpo de la mujer, de tal manera que en ese proceso de histerización ella viene a religarse a su propia finitud corporal en tanto útero reproductor.

Pero en esa misma operación de adscribir lo femenino al cuerpo, se da lo que podemos denominar la "nostalgia" de lo femenino. Porque de ese lado - y, por lo mismo, del lado del cuerpo- se asignan el cuidado, la interdependencia y los lazos comunitarios, que vienen a ser objeto de revalorización. Y si las mujeres han sido asimiladas a lo natural, a la tierra, a la inmanencia, a la corporalidad, se defiende ahora, desde el propio pensamiento feminista, poner todo eso en positivo en lugar de despreciarlo. De modo que la reclamación de la subjetividad femenina pasaría por la reclamación de la incardinación corporal; y el cuerpo se erige en lugar clave para la resignificación del sujeto feminista:

"La perspectiva que sugiero es que el punto de partida para las redefiniciones feministas de la subjetividad femenina es una nueva forma de materialismo, que pone el énfasis en lo incardinado y, por tanto, en la estructura sexualmente diferenciada del sujeto que habla. La variable de la sexualidad tiene una alta prioridad en el materialismo corporal por el que se aboga. En la teoría feminista una habla como una mujer, aunque el sujeto 'mujer' (...) no es una esencia definida de una vez por todas, sino que es más bien el lugar de un conjunto de experiencia múltiple, compleja y potencialmente contradictoria. 'Hablar como si' hace referencia a la 'política de la localización' de Adrienne Rich, es decir, a la incardinación como posicionalidad” (Braidotti, 1994: 199).

${ }^{2}$ Diccionario de la Lengua Española, Real Academia Española 
Pero, volvamos a las preguntas anteriores: ¿qué se está revalorizando al revalorizar el cuerpo como lugar de la subjetividad femenina? O, en otras palabras, ¿no se está proponiendo aquí una nueva realidad prediscursiva y, por lo mismo, esencializadora como portadora de la auténtica identidad femenina? En efecto, esta reflexión de Braidotti difícilmente puede casar con una posición constructivista que mantiene que

“el 'cuerpo' es en sí una construcción, como lo son los múltiples `cuerpos' que conforman el campo de los sujetos con género. No puede afirmarse que los cuerpos posean una existencia significable antes de la marca de su género; entonces, ¿en qué medida comienza a exisitir el cuerpo en y mediante la (s) marca(s) del género? ¿Cómo reformular el cuerpo sin verlo como un medio o instrumento pasivo que espera la capacidad vivificadora de una voluntad rotundamente inmaterial?” (Butler, 2007: 58)

Desde luego, parece que a simple vista una posición como ésta, que entiende el cuerpo como construcción radical, no tendría por qué estar reñida con una reivindicación del cuerpo, aun construido, como lugar de la subjetividad femenina. Pero la propia Butler deja clara la imposibilidad de tal combinación: carece de sentido hablar de una subjetividad dada y, mucho más, buscarle un lugar determinado y, en este caso, materialmente determinado previo a la acción:

"El argumento fundacionalista de la política de la identidad tiende a dar por sentado que una identidad primero debe ocupar su lugar para que se definan intereses políticos, y a continuación se inicie la acción política. Mi razonamiento es que no es preciso que exista un `agente detrás de la acción', sino que el 'agente' se construye de manera variable en la acción y a través de ella” (Butler, 2007: 278)

Estas posiciones de Butler han tenido un gran eco en el panorama del pensamiento feminista y también del feminismo en general. Sin embargo, es paradójico constatar que tal eco no obsta para que, también en el panorama actual, se vaya a la vez de la mano de la reivindicación del cuerpo. Curiosamente asistimos a un momento teórico donde ambos extremos se aceptan por igual, sin que se repare en la incompatibilidad profunda entre los mismos. Porque si excluimos todo topos identitario, dejará de tener sentido la apelación a la corporalidad femenina para su revaluación en positivo: nada habrá semejante a la privacidad, el cuidado, la inmanencia y otras características propias de ese cuerpo femenino que constituyan un lugar pre-discursivo en el que asentar identidad ni subjetividad algunas.

\section{2. ¿REVALORIZAR LA IDENTIDAD “MUJER=CUERPO”?}

La cuestión del cuerpo ha estado presente en el pensamiento filosófico desde la tradición platónica y aristotélica, hasta autores como Spinoza o Merleau-Ponty. Pero 
estos discursos hablan de un cuerpo neutro y universal, no marcado por la anatomía sexual y, en concreto, por la femenina:

"La mujer se ha identificado con su cuerpo más que el hombre-varón con el suyo. El hombre-varón ha intentado distinguirse de su propio cuerpo para reducirse a forma, a razón pura, desincorporada. La tradicional tendencia platónico-cristiana de reducir el ser humano a espíritu, a sustancia inteligible separada del mundo sensible y material, y menospreciar al cuerpo, reduciéndolo a simple instrumento del alma, es significativamente varonil y machista” (Pérez Estévez, 2008: 17-18)

Si el cuerpo ha sido lo olvidado, lo ocultado por la cultura moderna y su valoración de la mente incontaminada e higiénica (Heller y Féher, 1995: 17), puede decirse que la asociación entre el cuerpo y lo femenino ejemplifica paradigmáticamente esta reclusión en la sombra del discurso y del hacer públicos. De este modo, el cuerpo como lo femenino encarnado es sinónimo de lo doméstico, privado, de los vínculos familiares y comunitarios. ¿Se tratará de reclamar el lugar del cuerpo femenino como reclamación de todas estas esferas relegadas por la razón y los modos de vida desde la modernidad?

En su relectura de Bourdieu y Foucault, Susan Bordo concluye que el control social directo se ejerce sobre el cuerpo. Este control se ejerce a través de la práctica discursiva: "El cuerpo no es únicamente un texto de la cultura. Es también (...) un locus práctico y directo del control social” (Bordo, 2003: 165). De este modo el cuerpo femenino no existe fuera del discurso, porque es precisamente con ese cuerpo en particular con lo que la subjetividad empieza. Sin ánimo de enmendar la plana a esta consideración tan foucaultiana, sí cabría precisar que el control del cuerpo femenino, entendido como el control de lo femenino en tanto que cuerpo, también responde a prácticas no meramente discursivas: se impone por recursos de dominación tan materiales como también directos que, en casos límite, llegan incluso a la eliminación física, como lo analiza Federici pare el fenómeno histórico de la quema de brujas: represión de lo femenino y represión del cuerpo vienen así a resultar sinónimos.

En su análisis etnográfico "de las estructuras objetivas y de las formas objetivas de una sociedad histórica concreta, a la vez exótica e íntima, extraña y familiar, la de los bereberes de la Cabilia", para poder llevar a cabo "un trabajo de socioanálisis del inconsciente androcéntrico capaz de operar la objetivación de las categorías de ese inconsciente”, Pierre Bourdieu afirma

“Así pues, la definición social de los órganos sexuales, lejos de ser una simple verificación de las propiedades naturales, directamente ofrecidas a la percepción, es el producto de una construcción operada a cambio de una serie de opciones orientadas o, mejor dicho, a través de la acentuación de algunas diferencias o de la escotomización de algunas similitudes" (Bourdieu, 2000: 27). 
Y esta construcción social, que redunda en la diferencia, pasa a instituirse como dominación que adquiere la legitimación de lo objetivo:

"Gracias a que el principio de visión social construye la diferencia anatómica y que esta diferencia social construida se convierte en el fundamento y en el garante de la apariencia natural de la visión social que la apoya, se establece una relación de causalidad circular que encierra el pensamiento en la evidencia de las relaciones de dominación, inscritas tanto en la objetividad, bajo la forma de disposiciones objetivas, como en la subjetividad, bajo la forma de esquemas cognitivos que, organizados de acuerdo con sus divisiones, organizan la percepción de sus divisiones objetivas” (Bourdieu, 2000: 24).

Esta "construcción social naturalizada", en tanto manera de legitimar un orden, nuevo o establecido, que naturaliza la dominación, sólo es comprensible, sin embargo, si se parte de la previa legitimación de la necesidad de esa dominación: la necesidad de domesticar lo que es definido por sí mismo exceso indomable.

En su ensayo sobre La dominación masculina, que ya hemos utilizado aquí, Pierre Bourdieu habla de "la construcción social de los cuerpos" y sostiene que "El mundo social construye el cuerpo como realidad sexuada y como depositario de principios de visión y de división sexuantes” (Bourdieu, 2000: 22). Y esto es así porque "la división entre los sexos parece estar en el orden de las cosas, como se dice a veces para referirse a lo que es normal y natural, hasta el punto de ser inevitable" (Bourdieu, 2000: 21). Y casi al final de su obra, al hablar en su post-criptum sobre la dominación y el amor, Bourdieu habla de cómo el cuerpo queda fuera de los circuitos y de la lógica mercantilistas, razonando que

"la economía de los intercambios simbólicos, cuya forma suprema es el don de uno mismo, y del propio cuerpo, objeto sagrado, excluido de la circulación mercantil, y que, debido a que suponen y producen una relaciones duraderas y no instrumentales, se oponen diametralmente, como ha demostrado David Schneider, a los intercambios del mercado laboral, transacciones temporales, y estrictamente instrumental entre unos agentes no específicos, es decir indiferentes e intercambiables” (Bourdieu, 2000: 135).

Este cuerpo "excluido de la circulación mercantil", al que se le suponen "relaciones duraderas" podemos aquí asociarlo en particular con el cuerpo como sinónimo de feminidad. Se trata de ese cuerpo significado como lugar depositario de las aspiraciones a lo que no se somete al orden de relaciones transaccionales, un lugar que se crea "Incesantemente bajo la mirada de los demás", por el que "las mujeres están condenadas a experimentar constantemente la distancia entre el cuerpo real al que están encadenadas, y el cuerpo ideal al que intentan incesantemente acercarse” (Bourdieu, 2000: 87). 
De manera que no se trata sólo de establecer el cuerpo como instancia natural de división de los sexos; y, a partir de ahí, convenir en que "La diferencia biológica entre los sexos, es decir, entre los cuerpos masculino y femenino, $\mathrm{y}$, muy especialmente, la diferencia anatómica entre los órganos sexuales, puede aparecer de este modo, la justificación natural de la diferencia socialmente establecida entre los sexos y en especial de la división sexual del trabajo" (Bourdieu, 2000: 24). Además de como justificación natural de la diferencia "socialmente establecida", la diferencia biológico-anatómica registra una orientación unidireccional, por la que, en último término, sólo uno de los dos sexos es conceptualizado exclusivamente como cuerpo, en virtud de un discurso precisamente social, que requiere de su afuera para constituirse como hegemónico. Esto es tanto como decir que, si tal como mantiene Bourdieu, los cuerpos son percibidos en función de la posición social que los percibe, entonces habrá también que aventurar que tal percepción se hace, por así decirlo, de arriba abajo, o de dentro a afuera: es decir, desde la posición que domina al cuerpo dominado. Este "esquema postural o corpóreo"3, por el que captamos las relaciones externas en virtud de nuestro lugar en el mundo, hace que

"las propiedades corporales (sean) aprehendidas a través de los esquemas de percepción cuyo utilización en los actos de valoración depende de la posición ocupada en el espacio social: las taxonomía existentes tienden a enfrentar, jerarquizándolas, las propiedades más frecuentes en los dominadores $\mathrm{y}$ en los dominados (flaco/gordo, grande/pequeño, elegante/grosero, ligero/pesado, etc.” (Bourdieu, 2000: 84 -85).

Si esto es así, si la percepción depende de la posición social, parece que podemos interpretar que la percepción del cuerpo se instituye como tal para eso que queda fuera, sometido a la percepción que lo trasciende. Y, volviendo a nuestro argumento -y no alejándonos de la lectura de la propia Simone de Beauvoir-, diríamos por tanto que la percepción dominante masculina, abstraída de su propia corporalidad y finitud, realiza la autopercepción de sí como trascendencia a partir de la percepción de lo femenino como inmanencia corporal. Siguiendo a Bourdieu, creemos que no sólo se trata de que "La dominación masculina, que convierte a las mujeres en objetos simbólicos, cuyos ser (esse) es un ser percibido (percipi), tiene el efecto de colocarlas en un estado permanente de inseguridad corporal o, mejor dicho, de dependencia simbólica (. ..)”

\footnotetext{
${ }^{3}$ Tomamos esta expresión de Merleau-Ponty, en quien no se puede entrar aquí, ni sería pertinente hacerlo, pero a quien no podemos dejar de recordar, en particular cuando Bourdieu habla de la posición desde la que se percibe; en este sentido, Merleau-Ponty, Maurice (1999),Fenomenología de la percepción, Barcelona, Editorial Altaya; y (2003), El mundo de la percepción, México, Fondo de Cultura Económica.
} 
(Bourdieu, 2000: 86): se trata también, y esencialmente, de que esa percepción coloca a las mujeres en lo corporal mismo; en otras palabras, y parafraseando a Bourdieu, su ser consiste en ser cuerpo percibido.

¿Pero, qué significa "cuerpo" o qué queda asignado a éste? Al hablar de cuerpo hablamos de lo irracional, lo instintivo, de los vínculos originarios y primitivos, del lugar de la inmanencia, ... Y estas significaciones son posibles a partir del cuerpo mismo como construcción, como actos de significación en "la superficie del cuerpo":

"No obstante, cuando se entiende la identificación como una incorporación o fantasía hecha realidad queda claro que la coherencia es anhelada, esperada e idealizada, y que esta idealización es efecto de una significación corporal. En otras palabras, actos, gestos y deseo crean el efecto de un núcleo interno o sustancia, pero lo hacen en la superficie del cuerpo, mediante el juego de ausencias significantes que evocan, pero nunca revelan, el principio organizador de la identidad como una causa. Dichos actos, gestos y realizaciones -por lo general interpretados- son performativos en el sentido de que la esencia o la identidad que pretenden afirmar son invenciones fabricadas y preservadas mediante signos corpóreos y otros medios discursivos” ( Butler, 2007: 266).

Este constructivismo radical que, como ha señalado la propia Judith Butler, ha llevado a algunos de sus críticos a sospechar que alberga una cierta "somatofobia" (Butler, 2002: 30), no puede desentenderse de la identificación femenino=cuerpo, tal como se ha ido transmitiendo en la cultura y de generación en generación; e, incluso, cabría decir que lo ha hecho como efecto del patriarcado con la misma globalidad que éste tiene. Y desde tal constructivismo nada habría que reivindicar, pues nada hay previo a esa construcción que sea objeto de apelación:

"En cada cultura una operación simbólica básica otorga cierto significado a los cuerpos de las mujeres y de los hombres. Así se construye socialmente la masculinidad y la feminidad. Mujeres y hombres no son un reflejo de la realidad "natural", sino que son el resultado de una producción histórica y cultural, basada en el proceso de simbolización; y como 'productores culturales' desarrollan un sistema de referencias comunes” (Lamas, 2000: 4).

Sin embargo, puede que ya no sea útil seguir pensando el cuerpo desde el feminismo en los ejes de masculinidad-feminidad. O, como lo plantea Mari Luz Esteban, quizá la sexualidad no deba ser el único factor a tener en cuenta como el lugar donde se da la subversión en relación a lo corporal. Y tampoco dejan de ser problemáticas las versiones radicalmente constructivistas del cuerpo, al igual que las simplificadoramente esencialistas: pues el cuerpo sí "pesa", sino como forma de "ser", sí como forma de "estar", como acto o como práctica, que compone "itinerarios corporales”, que no por no constituir esencias sustantivas dejan de ser reales: 
"procesos vitales individuales pero que nos remiten siempre a un colectivo, que ocurren dentro de estructuras sociales concretas, y en los que damos toda la centralidad a las acciones sociales de los sujetos, entendidas éstas como prácticas corporales. El cuerpo es así entendido como el lugar de la vivencia, el deseo, la reflexión, la resistencia, la contestación y el cambio social, en diferentes encrucijadas económicas, políticas, sexuales, estéticas e intelectuales. Itinerarios que deben abarcar un periodo de tiempo lo suficientemente amplio como para que pueda observarse la diversidad de vivencias y contextos, así como evidenciar los cambios” (Esteban, 2004: 54).

Desde esta comprensión más amplia de cuerpo, que no lo reduce a la determinación sexual, pero que tampoco lo diluye en tanto mero constructo social, sino que lo piensa como "agente" (Esteban, 2004: 250), cabe preguntarse cómo podríamos concebir hoy el cuerpo desde los intereses feministas más actuales. Y de nuevo remitimos a la propuesta de Esteban, cuando afirma que, al igual que en el feminismo contemporáneo fue crucial la tarea de desvincular las categorías de mujer y madre, hoy habría que trabajar en el sentido de romper la identificación entre mujer y cuidadora. Pero, aclara, esta tarea no implica moverse en contra de un mundo que se base en el reconocimiento mutuo, la reciprocidad, el apoyo, la ayuda, o la solidaridad .

Por lo que venimos viendo, la identificación del cuerpo con lo femenino compone una de las caras más evidentes de un discurso que lo que persigue es subyugar a las mujeres, esto es, compone una dimensión esencial de un discurso de la dominación. Pero cabe o bien reclamar ese cuerpo femenino como lugar de la resistencia o del ser otro, o bien desvelar que se trata de una mera construcción que se disuelve en cuanto se la somete a un análisis deconstructivo.

Sin renunciar a un sano constructivismo, que siempre ha acompañado a la crítica feminista desde sus orígenes, aquí nos alineamos más bien con esa propuesta que, como se ha recogido aquí, señala el cuerpo como práctica, como "itinerario corporal", pero que no por eso deja de otorgarle un sentido (recordemos: económico, político, sexual, estético e intelectual). De manera que, si se trata de reivindicar el cuerpo femenino, se tratará de hacerlo en todas estas dimensiones, y no esencialmente como sexualidad, o como simple depositario de valores hetero-designados.

Por ello, al preguntarnos qué cuerpo reivindicar como feministas, habrá que preguntarse también en qué mundo queremos que tal cuerpo esté. Desde luego, no creo que se trate de reclamar sin más todo lo que la lógica masculina ha elaborado como femenino, y regresar con ello a una mujer-cuerpo definida por sus lazos domésticos y privados. Pero sí puede que, precisamente como feministas, estemos interesadas en un mundo más habitable, más regido por la lógica de la inter-dependencia, del apoyo mutuo, y no por una lógica mercantilista, deshumanizadora y letal para la vida. Pero estas reclamaciones dependen de las mentes, femeninas y no femeninas, que no son separables de sus cuerpos. Y las mentes femeninas, y con ello sus cuerpos, están sin duda más predispuestas a la posibilidad de otro mundo alejado del que hasta ahora ha sido y es producto de la mente sin cuerpo. 


\section{ALGUNAS REFLEXIONES PARA CONCLUIR}

La corporalidad diferenciada es transmitida, entre otras instancias y de manera muy central, por los modelos simbólicos que la escuela maneja. Porque es la escuela la encargada de reproducir la ideología y los estereotipos de género ya en la infancia. No sólo en cuanto a los contenidos, sino también en cuanto a las competencias, la escuela transmite a los niños y las niñas desde su más tierna infancia la identificación de género, masculina o femenina, en razón de la determinación de su corporalidad. Por tanto, una primera reflexión nos lleva a señalar que la escuela se convierte en uno de los lugares centrales en donde el sentido del cuerpo, su diferenciación y percepción, son transmitidos. Y esta transmisión activa la corporalidad diferenciada, masculinafemenina, como configuraciones de la propia identidad. En casos como la enseñanza de la educación física, la polarización entre cuerpos disímiles es especialmente reiterada, en tanto el ejercicio corporal masculino es orientado a un cuerpo sano para una mente sana, mientras que el ejercicio corporal femenino siempre se guía por desarrollar capacidades acordes con el embellecimiento del cuerpo, su estilización o se sentido de la actividad rítmica. Podría decirse, entonces, que en la tensión trascendenciainmanencia, a los niños se les educa el cuerpo que tienen, en tanto a las niñas se les disciplina el cuerpo que son.

Ya que la identidad sexual se constituye fundamentalmente a partir del cuerpo, este se convierte también en el dispositivo, en el sentido foucaultiano, que concentra simbólica y materialmente todos los contenidos socio-culturales por los que se conforman las identidades de género y la relación entre las mismas. Por ello, la escuela es ese lugar fundamental a la hora de disciplinar las identidades de género, a partir de la transmisión de las normas y dinámicas asociadas a la morfología sexual. Partiendo de aquí, cabe decir que la escuela, como socialización primaria que es, juega un papel determinante en mantener y reproducir el discurso del saber- poder sobre la corporalidad construida: el cuerpo es ese otro relato, que la escuela colabora activamente a consolidar y a reproducir.

Decir todo esto es tanto como decir, por supuesto, que el cuerpo también se enseña. Esto es, que no hay una realidad prediscursiva, como ya se decía más arriba, que conforme la sustancia corporal. Pero dicho esto, también cabe subrayar que la corporalidad, en tanto constructo cultural, pre-existe al sujeto y lo determina, de un modo material y simbólico a la vez.

Sin embargo, esta determinación corporal no es igual para hombres y mujeres. La corporalidad es una dimensión que el sujeto varón asume como una característica accidental de su ser: se le enseña desde pequeño a ir más allá de sus límites corporales y a vivirse a sí mismo en su dimensión universal. Incluso la morfología corporal juega en favor de ese despegarse masculino de la inmanencia, ya que el órgano genital masculino, en términos psicoanalíticos, es vivido por el sujeto a la vez como sí mismo y como ajeno, es el falo separado que propicia la conciencia del trascenderse y que, al mismo tiempo, encarna la trascendencia como parte constitutiva de la propias individualidad.

A las mujeres se les enseña, no a vivir atadas a su cuerpo, sino más bien a ser 
cuerpo. Parece que hay quien, desde supuestos feministas, pretendería reivindicar esta asociación de lo femenino al cuerpo como algo positivo: lo femenino vendría a leerse como el lugar donde es posible recuperar las formas de relación, el cuidado y la lógica no regida por las inter-relaciones mercantilistas. Esta, como hemos visto, es por ejemplo la propuesta de Silvia Federici. Esta es también la posición de otros feminismos insertos en una visión más comunitarista, o bien más defensora del pensamiento de la diferencia sexual.

Pero, una segunda reflexión nos conduce a concluir que, como se ha tratado de mostrar aquí desde una concepción preminentemente constructivista, esa estrategia corporalizadora no tiene sentido. Y no lo tiene por más de un motivo: por un lado, supone aceptar el relato de la modernidad cultural del dualismo mente (o razón)cuerpo, y la consiguiente relegación de las mujeres a lo segundo. Por otro lado, con esta tesis se acepta implícitamente un orden sustancial pre-discursivo, precisamente el cuerpo, que tiene que ser ahora entendido como realidad pre-existente al sujeto y a su conformación identitaria. Pero, cuál de estos dos relatos es menos asumible para una crítica feminista es una cuestión que parece contestarse por sí misma: ninguno. Ser cuerpo, para las mujeres, ha significado la medida de la opresión, de manera que no es razonable que la vía de la emancipación femenina pase por querer ser lo que se nos ha designado heterónomamente.

Con todo, una última reflexión que se impone es que no se trata de concluir la irrelevancia del cuerpo, ni de negar su materialidad. Se trata de pensar el cuerpo de otra manera y de disociarlo de la identificación con lo femenino. Porque, de otro modo, quedaríamos presas de ese discurso patriarcal que veta a las mujeres su capacidad de trascendencia. Una trascendencia que ha de estar guiada por los intereses en un mundo mejor, desde una lógica que aspire a no reproducir sin más las coordenadas dominantes de pensamiento. Se trata, en fin, de revalorizar para todos/todas una ética del cuidado y de la solidaridad, unas relaciones de inter-dependencia, que, más allá y más acá de su cuerpo, las mujeres conocen y pueden enseñar.

\section{REFERENCIAS BIBLIOGRÁFICAS}

BARRERA SÁNCHEZ, ÓSCAR (2011): "El cuerpo en Marx, Bordieu y Foucault”. Iberofórum.Revista de Ciencias Sociales de la Universidad Iberoamericana. Año VI, No. 11, 121-137

BEAUVOIR, SIMONE DE (1995): El segundo sexo, Vol. I: Los hechos y los mitos. Buenos Aires: Ediciones Siglo Veinte

BRAIDOTTI, ROSI (1994): Nomadic Subjects. Embodiment and Sexual Difference in Contemporary Feminist Theory. New York: Columbia University Press

BORDO, SUSAN: Unbearable Weight: Feminism, Western Culture and the Body. (Tenth Anniversary Edition), Los Angeles: California University

BOURDIEU, PIERRE (2000): La dominación masculina. Barcelona: Anagrama 
BUTLER, JUDITH (2002): Cuerpos que importan. Sobre los límites materiales y discursivos del "sexo". Buenos Aires-Barcelona: Paidós, 2002

BUTLER, JUDITH (2007): El género en disputa. El feminismo y la subversión de la identidad. Barcelona: Ediciones Paidós Ibérica, 2007

CIXOUS, HELENE (1995): La risa de la Medusa. Barcelona: Anthropos Editorial

ESTEBAN, MARI LUZ (2004): Antropología del cuerpo. Género, itinerarios corporales, identidad y cambio. Barcelona: Edicions Bellaterra

FEDERICI, SILVIA (2010): Calibán y la bruja. Mujeres, cuerpo y acumulación originaria, Madrid: Traficantes de Sueños

FOUCAULT, MICHEL (1978): Historia de la sexualidad. 1. La voluntad de saber, tomo I. Madrid: Siglo XXI

FOUCAULT, MICHEL (1986): Vigilar y castigar. Madrid: Siglo XXI Editores

HELLER, AGNES y FEHÉR, FERENC, (1995): La Modernidad y la liberación del cuerpo. Barcelona: Península

LAMAS, MARTA (2000): “Diferencias de sexo, género y diferencia sexual”. Cuicuilco, volumen 7, número 18, enero-abril, México 1-24

PÉREZ ESTEVEZ, ANTONIO (2008): “Tomás de Aquino y la razón femenina”, $R F$, vol.26, no.59, p.9-22

REVERTER BAÑON, SONIA (2001): “Modificación del cuerpo: ¿Parodia o subversión?”, Cuadernos Feministas, 5, Seminari d’Investigació Feminista, Universitat Jaume I, 51-58 